E3S Web of Conferences 4, 01004 (2014)

DOI: $10.1051 / \mathrm{e} 3$ sconf/20140401004

(C) Owned by the authors, published by EDP Sciences, 2014

\title{
The matter-wave laser interferometer gravitation antenna (MIGA): New perspectives for fundamental physics and geosciences
}

\author{
B. Canuel ${ }^{1,2, a}$, L. Amand ${ }^{1,3}$, A. Bertoldi ${ }^{1,2}$, W. Chaibi ${ }^{1,4}$, R. Geiger ${ }^{1,3}$, J. Gillot ${ }^{1,2}$, A. Landragin ${ }^{1,3}$, \\ M. Merzougui ${ }^{1,4}$, I. Riou ${ }^{1,2}$, S.P. Schmid ${ }^{1,2}$ and P. Bouyer ${ }^{1,2}$ \\ ${ }^{1}$ MIGA Consortium \\ ${ }^{2}$ LP2N, Laboratoire de Photonique Numérique et Nanosciences, Institut d'Optique Graduate School \\ IOA, Rue François Mitterrand, 33400 Talence, France \\ ${ }^{3}$ SYRTE - Observatoire de Paris, 77, avenue Denfert-Rochereau, 75014 Paris, France \\ ${ }^{4}$ ARTEMIS - Observatoire de la Côte d'Azur, Boulevard de l'Observatoire CS 34229, 06304 Nice \\ Cedex 04, France
}

\begin{abstract}
We are building a hybrid detector of new concept that couples laser and matter-wave interferometry to study sub Hertz variations of the strain tensor of space-time and gravitation. Using a set of atomic interferometers simultaneously manipulated by the resonant optical field of a $200 \mathrm{~m}$ cavity, the MIGA instrument will allow the monitoring of the evolution of the gravitational field at unprecedented sensitivity, which will be exploited both for geophysical studies and for Gravitational Waves (GWs) detection. This new infrastructure will be embedded into the LSBB underground laboratory, ideally located away from major anthropogenic disturbances and benefitting from very low background noise.
\end{abstract}

\section{Introduction}

Since its first demonstration [1] in 1991, Atomic Interferometry (AI) has shown to be an extremely performing probe of inertial forces. More recently, AI has revealed sensitivities to acceleration or rotation [2] competing with or even beating state-of-the art sensors based on other technologies. The high stability and accuracy of AI sensors relying on cold atoms is at the basis of several applications ranging from fundamental physics (e.g. tests of general relativity $[3,4]$ and measurements of fundamental constants [5-7]), geophysics (gravimetry [8], gradiometry [9]) and inertial navigation [10]. In contrast with these experiments, MIGA will explore the use of AI techniques to build a largescale matter-wave sensor which will open new applications in geoscience and fundamental physics. In contrast to standard AI based sensors, commonly limited by laboratory vibrations transmitted to the free falling atoms through the interrogation lasers, MIGA will exploit the superb seismic environment of a low noise underground laboratory [11] and will use suspended cavity mirrors for the definition of the

\footnotetext{
${ }^{a}$ Corresponding author: benjamin.canuel@institutoptique.fr
}

This is an Open Access article distributed under the terms of the Creative Commons Attribution License 4.0, which permits unrestricted use, distribution, and reproduction in any medium, provided the original work is properly cited. 


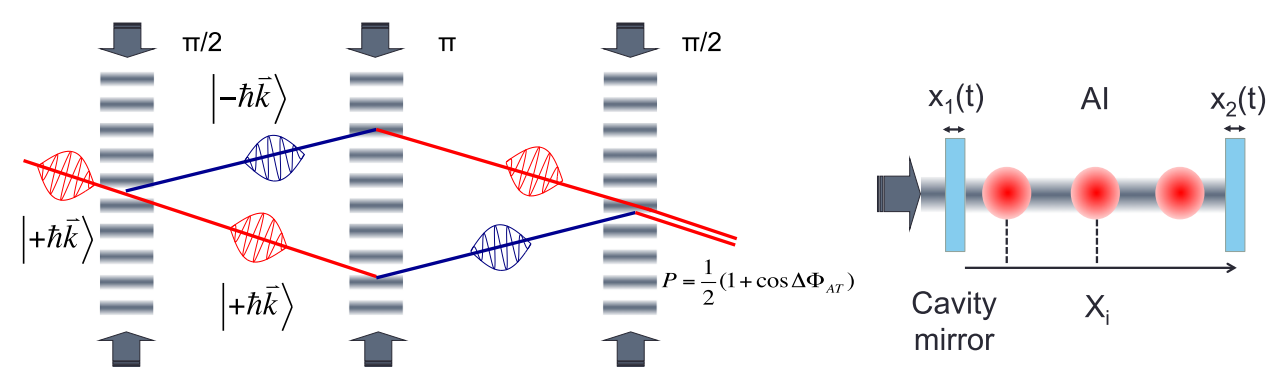

Figure 1. Left: principle of a Mach Zenhder interferometer for matter-waves. Right: geometry of the MIGA antenna combining a set of AIs interrogated inside an optical cavity.

interrogation field. This will provide a real breakthrough in sensitivity for the MIGA applications and for the development of next generation AI sensors.

MIGA will combine atom and laser interferometry techniques, manipulating an array of atomic ensembles distributed along the antenna by cavity enhanced Bragg pulses, so as to create an AI that will simultaneously read out motion of the cavity, GWs and inertial effects. The spatial resolution obtained with a set of three atomic sensors placed along the optical cavity will enable the separation of these contributions. This technique will bring to unprecedented sensitivities to gravity gradients fluctuations and open new perspectives for sub Hertz GW detection.

The sensitivity of the best working GW detectors based on giant optical interferometers $[12,13]$ are limited under a few tens of Hertz by several sources of cavity length noise that mimic the effects of GWs (like Newtonian noise, seismic noise, and radiation pressure noise). The MIGA interrogation scheme will allow to read GW signals free of cavity length noise, which opens new perspectives for ground based GW detection. In addition, MIGA will also provide measurements of gravity gradients fluctuations limited only by the AI shot noise, which will allow sensitivities of about $10^{-13} \mathrm{~s}^{-2} \mathrm{~Hz}^{-1 / 2}$ @ $2 \mathrm{~Hz}$. This instrument will then be capable to resolve $1 \mathrm{~m}^{3}$ of water a distances of about $100 \mathrm{~m}$ which opens important potential applications in geosciences.

In this paper we will first present the geometry of the MIGA detector and give details about its sensitivity to GWs and inertial signals. We will then present the design of the major sub-systems of the antenna such as atom interferometers, mirror suspensions and cavity control.

\section{MIGA geometry and sensitivity}

The AI geometry of MIGA is similar to the one of a Mach-Zenhder Interferometer for optical waves. This concept is described in Fig. 1-left where matter waves are manipulated by a set of counterpropagating laser pulses. At the entrance of the interferometer, a $\pi / 2$ pulse creates an equiprobable coherent superposition of states of the manipulated atoms. The matter-waves are then deflected by the use of $\pi$ pulse before being recombined with a second $\pi / 2$ pulse. To realize these beam-splitters and mirror pulses, MIGA will make use of Bragg diffraction of matter-waves on light standing waves [14]. Conservation of energy-momentum during this process imposes to couple only atomic states of momentum $|+\hbar k\rangle$ to state $|-\hbar k\rangle$ where $\mathrm{k}$ is the wave vector of the interrogation field. At the output of such interferometer, the transition probability between these states is obtained by a two waves interference formula $P=1 / 2\left(1+\cos \Delta \phi_{A T}\right)$. The atom phase shift $\Delta \phi_{A T}$ depends on the laser phase difference which is imprinted on the diffracted matter-wave during the interrogation process.

MIGA will make use of a set of such AIs interrogated by the resonant field of an optical cavity as described in Fig. 1-right. In this configuration, each AI will measures the inertial effects $s_{I}\left(X_{i}\right)$ along the cavity axis at position $\mathrm{X}_{i}$ together with $\mathrm{GW}$ effects associated to the cavity propagation of 


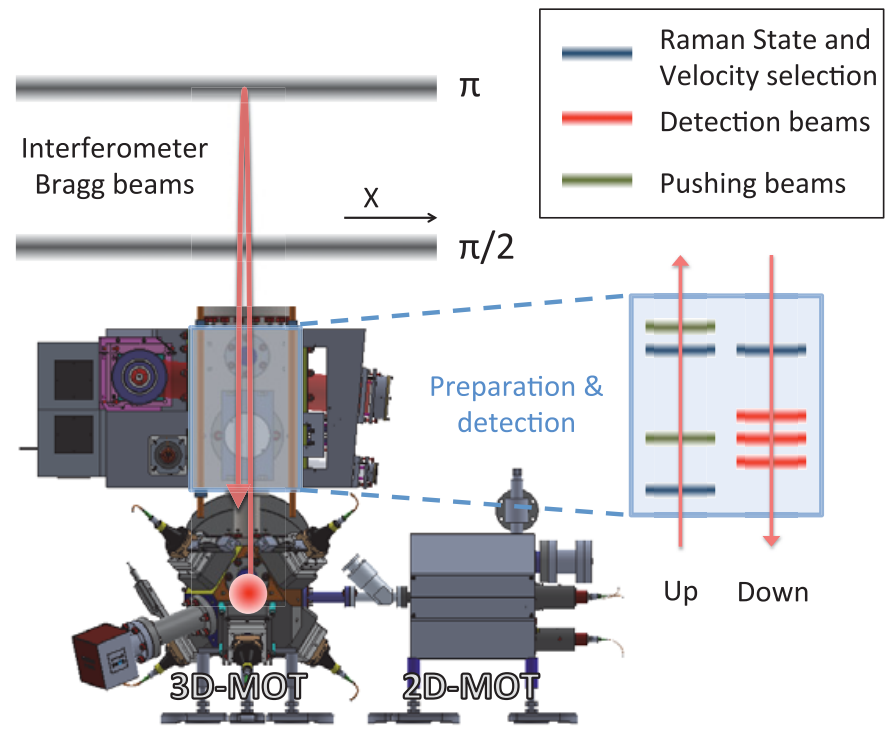

Figure 2. Description of the Atom head system.

the interrogation laser. Spurious effects such as fluctuation of cavity mirror position $\mathrm{x}_{1}(\mathrm{t})$ and $\mathrm{x}_{2}(\mathrm{t})$ or laser frequency noise $\delta v$ can also converts in phase noise of the resonant interrogation field and impact the response of each AI. Taking into account these different effects, the atom phase shift $\Delta \phi_{A T}\left(X_{i}\right)$ measured by the $\mathrm{AI}$ at position $\mathrm{X}_{i}$ will be written:

$$
\Delta \phi_{A T}\left(X_{i}\right)=\frac{4 \pi v_{0}}{c} s_{x_{2}}+\frac{4 \pi}{c}\left[-s_{\Delta v}+\frac{\nu_{0}}{2} s_{h}\right]\left(X_{i}-L\right)+s_{I}\left(X_{i}\right)
$$

where $v_{0}$ is the laser frequency, $h$ is the strain modification induced by GW, L is the mean cavity length and $\mathrm{s}_{u}$ accounts for the ponderation of the time-fluctuations of effect $u$ by the sensitivity function $\mathrm{s}$ of the AI [15]: $s_{u}=\int_{-\infty}^{\infty} s(t) \frac{d u(t)}{d t} d t$. Common mode rejection between AI signals will enable to cancel out most of the contribution of cavity mirror position fluctuations and influence of laser frequency noise will be kept under ultimate measurement noise by using state-of-the-art ultra stable laser techniques.

Then, to separate between Inertial and GW signals of Eq. (1) we will use the spatial resolution that is offered by the set of AIs placed along the cavity. Indeed, GW effects are seen as gradients while inertial contribution has spatial signature.

\section{MIGA antenna}

In this section we detail two of the most important systems of the MIGA antenna, the Atom Interferometers which takes care of preparation and detection of the cold atom sources and the cavity suspension and control which is crucial for the stability of the interrogating laser field.

\subsection{Atom interferometers}

The atomic heads system takes care of delivering the sources of cold atoms interrogated inside the MIGA cavity and is presented in Fig. 2. It consists in the cooling and preparation of a cold atom source launched on a quasi-vertical trajectory. After interrogation by a set of Bragg interrogation pulses inside the cavity, the atoms fall back towards the detection which is also part of the system. 


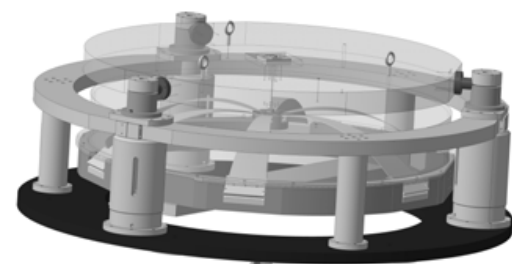

Figure 3. Scheme of suspension system of the MIGA payloads.

Preparation of the $\mathrm{Rb} 87$ atomic source used is performed as follows. A cold atom beam produced from a Rubidium vapor at few $10^{-9} \mathrm{mBar}$ is produced using a combination of laser beams and magnetic fields in a 2D Magneto Optical Trap (2D-MOT). These atoms are pushed towards the trapping region of a different vacuum chamber where they are further cooled in a 3D Magneto Optical Trap (3D-MOT), down to the sub-Doppler regime corresponding to a temperature of few $\mu \mathrm{K}$. Detuning the top lasers of the 3D-MOT with respect to the bottom lasers then creates a conveyor for the atoms in order to launch them vertically at a velocity of $\approx 4 \mathrm{~m} / \mathrm{s}$.

In order to optimize the contrast of the atom interferometer, the quantum state of the atoms is prepared on their way up, before the interrogation region. A first counter-propagating velocity-selective Raman pulse is used to select the atoms in the $\mathrm{m}_{\mathrm{F}}=0$ Zeeman sub-level of the $\mathrm{F}=2$ hyperfine state, with a relatively narrow velocity class (width of 1 photon recoil, corresponding to a temperature of $300 \mathrm{nK}$ in the direction ( $\mathrm{X}$ axis) of the Raman lasers). The unselected atoms are then pushed by a laser tuned on resonance with the cycling transition. This procedure is repeated a second time to clean the remaining unwanted atoms produced by spontaneous emission on the first Raman selection pulse.

After their interrogation by the Bragg beams in the atom interferometer, the two different momentum states of the atoms are resolved using Raman laser labeling of the two different hyperfine states of the $\mathrm{Rb} 87$ atoms $(\mathrm{F}=1$ and $\mathrm{F}=2)$. Detection of the atoms projected in $\mathrm{F}=2$ is first realized with a light beam tuned on resonance on the $\mathrm{F}=2 \rightarrow \mathrm{F}^{\prime}=3$ transition. The remaining $\mathrm{F}=1$ atoms are re-pumped to the $\mathrm{F}=2$ state and counted as before in the third light sheet. Two-quadrant photodiodes provide the fluorescence signal used to reconstruct the normalized atomic populations an then deduce the transition probability and the response of the AI, $\Delta \phi_{A T}$.

\subsection{Cavity suspension and control}

As shown in Eq. (1), ground seismic noise can impact the AI response through phase modulation of the interrogation field due to the cavity mirror motion. Indeed, seismic noise along cavity direction may directly modify the relative phase between the interrogation beams. Along other degrees of freedom it may recouple noise mainly through fluctuations of the intracavity wavefront. Although most of the seismic noise will be cancelled out by common mode rejection between different AIs, this technique has some limitations due to inhomogeneities of the AI response, determined for example by mode diffraction and light propagation delays.

To reduce even further the impact of seismic noise, MIGA uses mirrors isolated with mechanical filters. The overall architecture of this system is similar to the so-called super-attenuators developed for the VIRGO interferometer [16]. The MIGA isolation system can be been seen in Fig. 3, it consists in two $20 \mathrm{~cm}$ pendulums for horizontal isolation and one vertical isolator made from pre-constrained blades.

Compared to the VIRGO system, the MIGA vertical isolator differs for the addition of a hinge used to center the blades. This guarantee a better symmetry to the system which reduces the verticalhorizontal coupling. The blades are also extended to reduce the maximum equivalent stress below 50\% of the Maraging alloy elastic limit. 

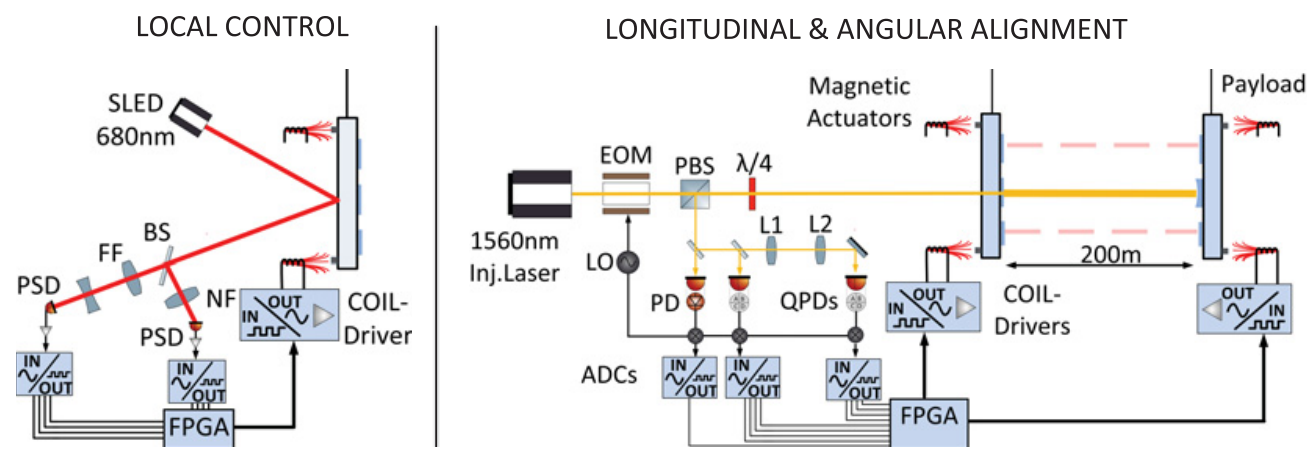

Figure 4. Description of the mirror Local Control and scheme of the longitudinal and angular alignment system.

In order to control the payload alignment at low frequency without reintroducing seismic noise from the ground, the control of the cavity mirors will be achieved using magnetic actuation. Four different magnet-coil actuators will be used for each payload, allowing angular and longitudinal control of the cavity. The control system of the payload is split into two independent parts. A first system, called local control, provides an independent, absolute and large dynamic control of each payload alignment. The second system, called linear alignment, is a relative, high precision control system used for an active control of the cavity alignment when it is close to resonance.

The scheme of the local control system is shown in Fig. 4-left. It enables to sense shift and tilts of the payload with respect to two Position Sensing Devices (PSDs) rigidly fixed from the ground. The local control system has to comply with the resonance frequencies associated with the payload's different degrees of freedom. Moreover the gain of each of the 4 magnet-coil-actuators are slightly different which leads to a cross-talk for example between shifts and rotations. To overcome these problems an actuation matrix will be implemented in a FPGA-based central control system. This central FPGA is directly connected to the 16-bit inputs of the coil's current-drivers.

To sense the different degrees of freedom, the light of a Superluminescent Diode (SLED / $680 \mathrm{~nm}$ ) is shined on the back of each payload under a large angle (about $45^{\circ}$ ) and an optical setups enables to sense on two different PSDs the shifts (Near Field-NF) and the tilts (Far Field-FF) [17] of the payloads. Both PSD signals are then digitized and given to the central FPGA for control.

Once the payloads are roughly aligned thanks to the local controls, the Ward-Method [18] is used to reduce alignment fluctuations of the cavity while the Pound-Drever-Hall (PHD) technique [19] takes care of the longitudinal control following a scheme represented in Fig. 4-right. As the $780 \mathrm{~nm}$ interrogation beams are time-modulated to generate the Bragg pulses and cannot be used for control purposes, this system makes use of a coherent $1560 \mathrm{~nm}$ beam also resonating inside the cavity. This laser light is phase modulated by an EOM before being sent to the cavity. Light reflected from the cavity goes to a set of demodulated photodiodes that provides the error signals. For the alignment, a set of two quadrants photodiodes placed at different Gouy phases are needed. All photodiodes signals are then digitized and given to the central FPGA which uses the same control-path as before to correct the position of the payloads.

\subsection{Conclusion}

We have presented the status of MIGA, an experiment under construction where laser and matterwave interferometry will be interwined to monitor the evolution of the gravitational field at very high sensitivity. Given its ultra-low noise underground location and its $200 \mathrm{~m}$ long probe arm, the experiment will be used to investigate both geophysical phenomena and sub-Hertz variations of the strain tensor of 
space-time, with a novel approach in the quest for gravitational waves. The experimental realization is based on a modular design, here described in its main components: several atom interferometer heads are simultaneously interrogated using Bragg pulses enhanced with an optical cavity; the cavity is held in ultra-high-vacuum and the mirrors require both a suspension and a control system to limit the effect of seismic noise on the gravity measurements.

\section{References}

[1] P.R. Berman, Atom Interferometry (Academic Press, 1997).

[2] B. Canuel, F. Leduc, D. Holleville, A. Gauguet, J. Fils, A. Virdis, A. Clairon, N. Dimarcq, Ch.J. Bordé, A. Landragin, and P. Bouyer, Phys. Rev. Lett. 97, 010402 (2006).

[3] S. Dimopoulos, P.W. Graham, J.M. Hogan, and M.A. Kasevich, Phys. Rev. Lett. 98, 111102 (2007).

[4] D.N. Aguilera, et al., Class. Quantum Grav. 31, 115010 (2014).

[5] J.B. Fixler, G.T. Foster, J.M. McGuirk, and M.A. Kasevich, Science 315, 74 (2007).

[6] G. Lamporesi, A. Bertoldi, L. Cacciapuoti, M. Prevedelli, and G.M. Tino, Phys Rev. Lett. 100, 050801 (2008).

[7] R. Bouchendira, P. Cladé, S. Guellati-Khelifa, F. Nez, and F. Biraben, Phys Rev. Lett. 106, 080801 (2011).

[8] A. Peters, K.Y. Chung, and S. Chu, Nature 400, 849 (1999).

[9] M.J. Snadden, J.M. McGuirk, P. Bouyer, K.G. Haritos, and M.A. Kasevich, Phys Rev. Lett. 81, 971 (1998).

[10] M.A. Kasevich and B. Dubetsky, United States Patent 7317184.

[11] T. Farah, C. Guerlin, A. Landragin, P. Bouyer, S. Gaffet, F. Pereira Dos Santos, and S. Merlet, arXiv:1404.6722.

[12] F. Acernese, et al., Journal of Optics A: Pure and Applied Optics 10, 064009 (2008).

[13] B.P. Abbott, et al., Reports on Progress in Physics 72, 076901 (2009).

[14] P.J. Martin, B.G. Oldaker, A.H. Miklich, and D.E. Pritchard, Phys. Rev. Lett. 60, 515-518 (1988).

[15] P. Cheinet, B. Canuel, F. Pereira Dos Santos, A. Gauguet, F. Yver-Leduc, and A. Landragin, Instrumentation and Measurement, IEEE Transactions on 57 , 1141-1148 (2008).

[16] The VIRGO collaboration, Astroparticle Physics 33, 182-189 (2010).

[17] B. Canuel, E. Genin, M. Mantovani, J. Marque, P. Ruggi, and M. Tacca, Appl. Opt. 53, 2906-2916 (2014).

[18] E. Morrison, B.J. Meers, D.I. Robertson, and H. Ward, Appl. Opt. 33, 5041-5049 (1994).

[19] R.W.P. Drever, Appl. Phys. B 31 , 97 (1983). 